\title{
Assessment of mesial temporal sclerosis through MRI processing
}

Castillo , D., Samaniego, R., Jiménez, Y., Sánchez, A., Carrión, J., et al.

D. Castillo , R. Samaniego, Y. Jiménez, A. Sánchez, J. Carrión, M. J.

Rodríguez Álvarez, "Assessment of mesial temporal sclerosis through MRI processing," Proc. SPIE 11469, Emerging Topics in Artificial Intelligence 2020, 114690W (20 August 2020); doi: 10.1117/12.2569043

SPIE. Event: SPIE Nanoscience + Engineering, 2020, Online Only 


\title{
Assessment of mesial temporal sclerosis through MRI processing
}

\author{
D. Castillo *a,c, R. Samaniego ${ }^{\mathrm{b}}$, Y. Jiméneze ${ }^{\mathrm{a}, \mathrm{c}}$, A. Sánchez ${ }^{\mathrm{a}}$, J. Carrión ${ }^{\mathrm{a}}$, M. J. Rodríguez-Álvarez ${ }^{\mathrm{c}}$ \\ ${ }^{a}$ Chemistry and Exact Sciences Department, Universidad Técnica Particular de Loja, Ap. 11-01- \\ 608, Loja-Ecuador; ${ }^{b}$ Dept. of Radiology, Hospital UTPL, Loja-Ecuador; ${ }^{c}$ Instituto de \\ Instrumentación para Imagen Molecular (i3M) \\ Universitat Politècnica de València - Consejo Superior de Investigaciones Cientificas (CSIC), \\ Valencia, Spain
}

\begin{abstract}
Mesial temporal sclerosis (MTS) is the principal cause of complex epilepsy, is manifested principally by gliosis and hippocampal volume loss. This project aims to develop an algorithm that allows automatic measurement of hippocampal volume and signal intensity in magnetic resonance imaging. The algorithm developed uses preprocessing of the images to reduce the artifacts and for the extraction of the features were used techniques of machine learning (support vector machine) and texture analysis. Results can help to optimize time in the assessment of the mesial temporal sclerosis and can contribute to the best training to the youngers neuroradiologists.
\end{abstract}

Keywords: machine learning, brain MRI, Mesial temporal sclerosis, hippocampal volume, epilepsy

\section{INTRODUCTION}

Neuroimaging is a highly interdisciplinary field of research in the health and medicine sciences. Different works manifest the fundamental role of the neuroimaging in the determination of neurological diseases and disorders and the support that offer to neuroradiologist in giving an accurate diagnosis, prognosis, and monitoring, due also the neuroimaging has been recognized as a powerful tool to analyze brain changes. ${ }^{1-9}$ Inside of this field, there are different modalities: e.g. for to analyze of the structure of the brain the Computed Tomography (CT) and Magnetic Resonance images (MRI) are the most used; and for to do a physiological analysis was used the Positron emission tomography (PET), single-photon emission tomography (SPECT) and functional MRI for to do a brain analysis of images ${ }^{10,11}$.

The MRI is widely used in the brain images segmentation and their posterior analysis due it offers high-resolution images of the anatomical structures and is a noninvasive safe method for the patients ${ }^{1,3,8}$. In this sense, Yamanakkanavar et al. ${ }^{8}$ shows that a detailed analysis of the tissue structures from segmented MRI leads to more accurate classification of specific brain disorders ${ }^{8}$, but at the same time indicates that the manual segmentation is a very laborious and subjective task to that consuming time and also requires a deep knowledge of the brain anatomy ${ }^{8}$. That is one of the reasons that in the last years there are continuous research and advances, especially in the field of machine learning (ML) and deep learning (DL) to develop automatic algorithms for brain segmentation and detection of brain disorders; even though, the automatic medical segmentation continues to be a complex and challenging problem ${ }^{6,12,13}$.

Among the pathologies and neurological disorders are the epilepsies. According to Raghavendra et al. ${ }^{13}$, the definition of epilepsy is " $a$ disorder of the brain characterized by enduring predisposition to generate the epileptic seizures." 13 It affects both male and female sexes of all ages. ${ }^{13}$ Magnetic resonance imaging (MRI) is one of the most important tools that permit to determine the syndromic diagnosis and possible etiology of epilepsy ${ }^{11}$. Concerning the pathological findings in patients with epilepsy are mesial temporal sclerosis (MTS), vascular anomalies, low-grade glial neoplasm, and malformations of cortical development ${ }^{11}$.

*dpcastillo@utpl.edu.ec; phone+593 07370 1444; ext.3204; www.utpl.edu.ec

Emerging Topics in Artificial Intelligence 2020, edited by Giovanni Volpe, Joana B. Pereira,

Daniel Brunner, Aydogan Ozcan, Proc. of SPIE Vol. 11469, 114690W · (c) 2020 SPIE

CCC code: $0277-786 X / 20 / \$ 21 \cdot$ doi: $10.1117 / 12.2569043$

Proc. of SPIE Vol. 11469 114690W-1 
Nataraja et al ${ }^{14}$ mention that despite epilepsy is well manageable with antiepileptic drugs, there are approximately $30 \%$ of epilepsy patients who are not responding to optimal treatment. In this sense, Azab et al. ${ }^{15}$ remark that the mesial temporal sclerosis (MTS) is the "most common pathologic entity encountered in epilepsy surgery series"

Mesial temporal sclerosis (MTS) also called Hippocampal sclerosis (HS $)^{11}$ is "characterized by neuronal loss and chronic fibrillary gliosis on the pyramidal cell layer of the hippocampus"14,16. Yamanakkanavar et al. ${ }^{8}$ and Azab et al. ${ }^{15}$ indicate that the MR imaging is the best technique of imaging preoperative that helps to detect and confirm the presence of the MTS, which is due the clinical MRI can detect the gross hippocampal atrophy changes. This changes in the hippocampal are appreciated in a visual way for the radiologists; however, this task is more reliable if is do it by experienced radiologist due to the subtlety of the changes ${ }^{15}$.

Silva et al. ${ }^{16}$ in their work show that the rate of detection of MTS by visual inspection on MRI is variable between $62 \%$ and $85 \%{ }^{15-17}$ and this assessment is in the function of the experience of the radiologist and the degeneration of the hippocampal structures. The modalities of MR imaging used to detect these abnormalities and evaluate the shape, orientation, volume and internal structure of the hippocampal anatomy are the high resolution T1-weighted with inversion recovery (IR), T2-weighted, and the fluid-attenuated inversion recovery (FLAIR), especially to assess signal intensity ${ }^{11,14,16}$.

There are some works ${ }^{12,13,15,16,18-25}$ that use different techniques of machine learning and deep learning such as support vector machine(SVM), artificial neural network (ANN), convolutional neural networks (CNN); illustrate different results to develop an automatic assessment of the volumetry of the hippocampus and the signal intensity of the abnormalities that characterize the MTS. However, for all these authors, like indicate Yamanakkanavar et al. ${ }^{8}$ "the segmentation of the hippocampus, is a difficult task due to its small size and volume, the anatomical variability, low signal to noise ratio(SNR), intensity inhomogeneities. ${ }^{" 1,19,26}$, although it is an important biomarker for the computeraided diagnosis (CAD) systems.

In this context, the principal aim of this work is to develop an algorithm that will be able to assess the principal features to detect the MTS, hippocampal volume loss, and hippocampal hyperintensity, using especially the coronal images T2 and FLAIR modalities of MRI. The algorithm proposed has different phases: collect images (dataset), preprocessing images, and processing images to determine the principal features and region of interest (ROI) using techniques of machine learning, like support vector machine (SVM), principal component analysis (PCA), and analysis of textures. This paper presents an advance of the algorithm, due that requires the validation of the experienced and experts radiologists and the posterior evolution for uses techniques of deep learning with a larger dataset. The paper is organized as follows: section two indicates the materials (description of the dataset) and the methodology used for the algorithm; in section three present the results and finally the section four gives a brief line of conclusion and discussion of the results obtained.

\section{MATERIALS AND METHODS}

The schema in the figure 1 ilustrates the architecture and the methodology of the algorithm proposed with its different steps: the dataset preprocessing, feature extraction, feature selection, and classification.

\subsection{Data acquisition}

The collection of images for uses in the algorithm is formed by:

- 30 healthy patients adults (both males and females) and;

- 70 patients with some disorder (45 have findings of mesial temporal sclerosis, 15 patients have other lesions such as ischemia or stroke, and 10 patients have brain tumors)

All studies series have been anonymized before collected from HUTPL and other different hospitals in Ecuador. 
The images T1-weighted, T2-weighted, and FLAIR images were acquired by the equipment of RM Philips Medical Systems, model Achieva 1.5 Teslas.

\subsection{Preprocessing}

In the preprocessing stage, we use python scripts to exclude the possible artifacts presented into the images, especially into the temporal-mesial structures in the coronal FLAIR images ${ }^{25}$. Figure 2 shows an example of possible artifacts in the FLAIR images in the temporal-mesial structures.

\subsection{Feature extraction and feature selection}

Feature extraction consists of identifying the brain regions, which will be the principal characteristics of the object of interests ${ }^{27}$ and in a posterior, the features most relevant will be the input for the classification.

The extraction of features was done in agreement with the expert neuroradiologist. Once that the images are free of artifacts and selected the region of interest (ROI), the anatomical structures that involve the hippocampus (temporalmesial) will be described according to the features of intensity, shape, and texture analysis. The features to extract after the get the ROIs are in concordance with ${ }^{27}$ : Shape Features of the hippocampus; volume, area, perimeter, irregularity, shape index. Intensity and signal features; mean, variance, standard variance, median intensity, skewness, and Kurtosis.Texture features concerning the hippocampus also; contrast, correlation, entropy, energy, homogeneity, the sum of square variance.

\subsection{Classification}

During the feature extraction and feature selection, each image was transformed into a column vector of features, and each value corresponded to a single corresponding voxel intensity. Feature extraction allows the original data to transform into analyzable input data ${ }^{28}$. In this stage of the algorithm, we use the SVM classifier and combination with the principal component analysis (PCA).

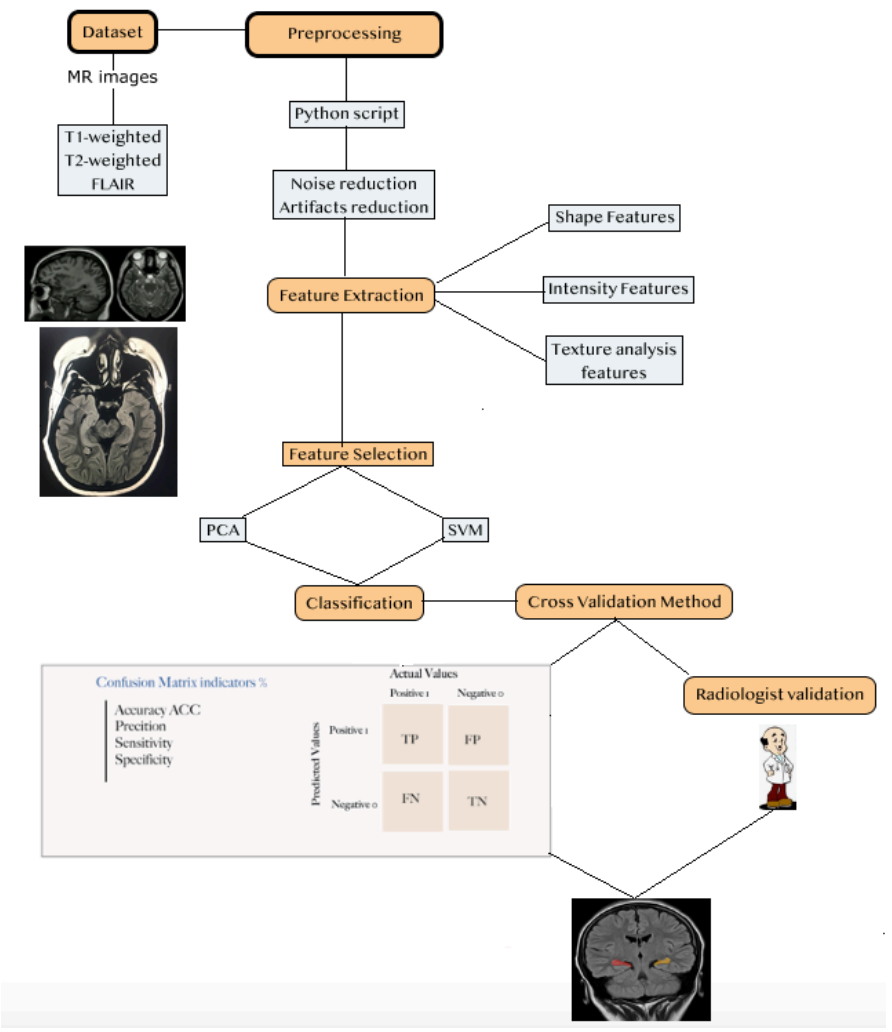

Figure 1. The architecture of the different stages of the algorithm proposed. The first stage consists of the collection of the dataset, preprocessing the images, and feature extraction. The second stage is feature selection, classification, and validation. 


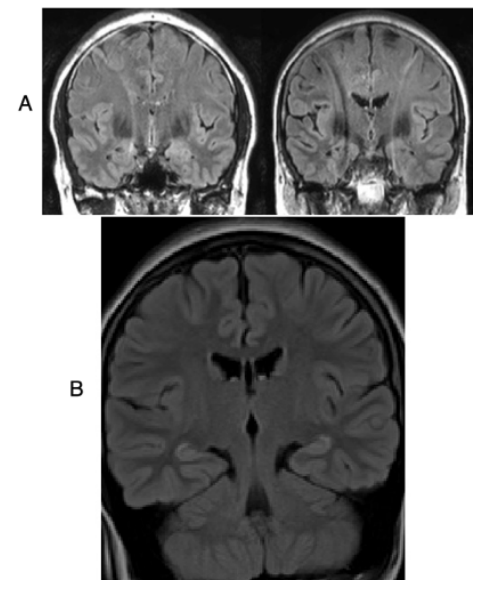

Figure 2. FLAIR coronal images of the structural anatomy of the brain: section (A) shows a couple of images into the zone of the temporal-mesial structures; and section (B) shows an image without artifacts. Figure adapted from Huppertz et al. ${ }^{25}$

\section{RESULTS}

In this work only is reported the results derivates from the first stage of the algorithm proposed: the dataset collection, preprocessing and the analysis of the features extraction through the definitions of ROIs and the data for volume hippocampus and intensity signal. In the next work will be reported an analysis exhaustive of the parameters concerning the feature selection and classification.

Figure 3 shows that is possible to do a segmentation of the hippocampus to determine the volume using texture analysis and machine learning techniques. Specifically, in the figure (B1) and (B2) we can see a level of asymmetric in the volume and a hyperintense signal in the lobe on the right side, consequently, these findings show that this patient has mesial temporal sclerosis in a low level. Also in figure 3 in (C), it is presented a segmentation of the hippocampus from a sagittal and cortical view.

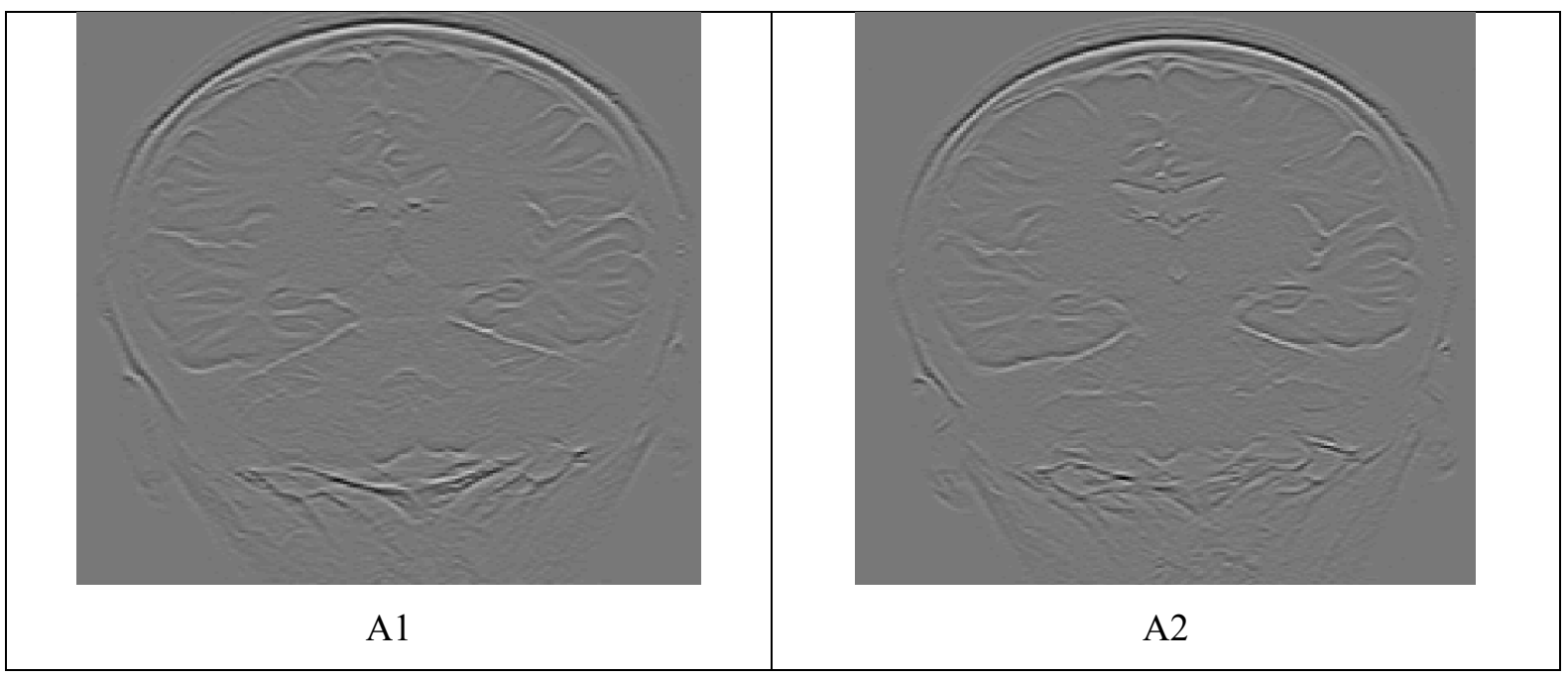




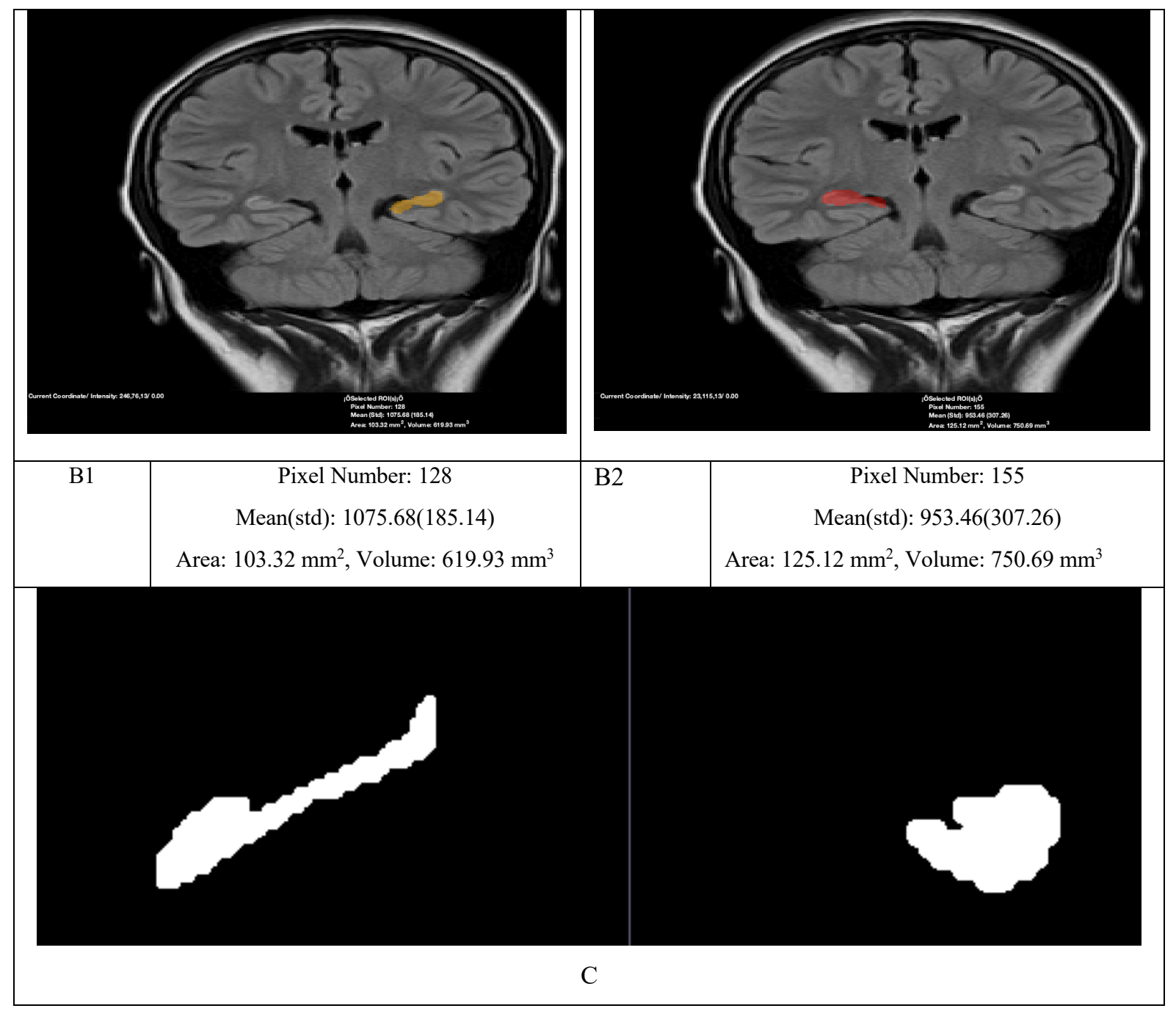

Figure 3. (A1) y (A2) shows the textures analysis of the series for each patient, this analysis allows to us to do a better identification of the anatomical structures that involve the MTS. (B1) and (B2) shows the segmentation and the values of the features of shape from the hippocampus, it is shown the volume for the left and right side of the lobe temporal; we can see a level of asymmetry in the volume and a hyperintense signal in the lobe on the right side, onsequently these findings show that this patient has mesial temporal sclerosis (MTS) in a low level. (C) The structure of the hippocampus segmented from a sagittal and cortical view.

\section{CONCLUSIONS AND DISCUSSIONS}

The results shown confirm that is is possible to get a good criterion for determining the MTS through the find the automatic volume hippocampus and the variation of the signal and intensity in the anatomical structures. It is in agreement with Huppertsz et $\mathrm{al}^{25}$., Yamanakkanavar et $\mathrm{al}^{8}$., Silva et $\mathrm{al}^{16}$, Nataraja et $\mathrm{l}^{14}$ and Cendes et $\mathrm{al}^{11}$ in which works present also different methods of segmentation and determination automatic of the hippocampus and the signal intensity. However, the gold standard for determining the MTS is yet the visual analysis and the experience ${ }^{15}$; for that reason, in the next work, we will report the analysis of the signal and the volume of the hippocampus with a more rate of training and reliable using deep learning techniques. 


\section{REFERENCES}

[1] Tillema, J.-M. and Pirko, I., "Neuroradiological evaluation of demyelinating disease," Ther Adv Neurol Disord 6(4), 249-268 (2013).

[2] Castillo, D., Samaniego, R., Jiménez, Y., Cuenca, L., Vivanco, O. and Rodríguez-Álvarez, M. J., "Magnetic resonance brain images algorithm to identify demyelinating and ischemic diseases," Proc SPIE Int Soc Opt Eng 10752, Tescher A.G., Ed., SPIE (2018).

[3] Castillo, D., Samaniego, R., Jiménez, Y., Cuenca, L., Vivanco, O. and Rodríguez-Álvarez, M. J., "Demyelinating and ischemic brain diseases: Detection algorithm through regular magnetic resonance images," Proc SPIE Int Soc Opt Eng 10396, Tescher A.G., Ed., SPIE (2017).

[4] Castillo, D. P., Samaniego, R. J., Jimenez, Y., Cuenca, L. A., Vivanco, O. A., Alvarez-Gomez, J. M. and Rodriguez-Alvarez, M. J., "Identifying Demyelinating and Ischemia brain diseases through magnetic resonance images processing," presented at 2019 IEEE Nuclear Science Symposium and Medical Imaging Conference, NSS/MIC 2019, 2019.

[5] BOWMAN, F. D., "Brain Imaging Analysis," Annu Rev Stat Appl 1, 61-85 (2014).

[6] Akkus, Z., Galimzianova, A., Hoogi, A., Rubin, D. L. and Erickson, B. J., "Deep Learning for Brain MRI Segmentation: State of the Art and Future Directions," J Digit Imaging 30(4), 449-459 (2017).

[7] Panayiotopoulos, C. P., [Brain Imaging in the Diagnosis and Management of Epilepsies], Bladon Medical Publishing (2005).

[8] Yamanakkanavar, N., Choi, J. Y. and Lee, B., "MRI Segmentation and Classification of Human Brain Using Deep Learning for Diagnosis of Alzheimer's Disease: A Survey," Sensors (Basel) 20(11) (2020).

[9] Sidhu, M. K., Duncan, J. S. and Sander, J. W., "Neuroimaging in epilepsy," Current Opinion in Neurology 31(4), 371-378 (2018).

[10] Powers, W. J. and Derdeyn, C. P., "Neuroimaging, Overview," [Encyclopedia of the Neurological Sciences (Second Edition)], M. J. Aminoff and R. B. Daroff, Eds., Academic Press, Oxford, 398-399 (2014).

[11] "Neuroimaging of epilepsy.", <https://www.ncbi.nlm.nih.gov/pmc/articles/PMC5256664/> (12 August 2020 ) .

[12] Sharma, N. and Aggarwal, L. M., “Automated medical image segmentation techniques," J Med Phys 35(1), 3-14 (2010).

[13] Raghavendra, U., Acharya, U. R. and Adeli, H., "Artificial Intelligence Techniques for Automated Diagnosis of Neurological Disorders," ENE 82(1-3), 41-64 (2019).

[14] Nataraja, V., Farook, A. S., Elangovan, V., Ahmed, A. and Magudeeswaran, P. K., "Magnetic Resonance Imaging Evaluation of Hippocampus with T2 Relaxation Time," 5 (2017).

[15] Azab, M., Carone, M., Ying, S. H. and Yousem, D. M., "Mesial Temporal Sclerosis: Accuracy of NeuroQuant versus Neuroradiologist," AJNR Am J Neuroradiol 36(8), 1400-1406 (2015).

[16] Silva, G., Martins, C., Moreira da Silva, N., Vieira, D., Costa, D., Rego, R., Fonseca, J. and Silva Cunha, J. P., "Automated volumetry of hippocampus is useful to confirm unilateral mesial temporal sclerosis in patients with radiologically positive findings," Neuroradiology Journal 30(4), 318-323 (2017).

[17] Coan, A. C., Kubota, B., Bergo, F. P. G., Campos, B. M. and Cendes, F., "3T MRI quantification of hippocampal volume and signal in mesial temporal lobe epilepsy improves detection of hippocampal sclerosis," AJNR Am J Neuroradiol 35(1), 77-83 (2014).

[18] Despotović, I., Goossens, B. and Philips, W., "MRI Segmentation of the Human Brain: Challenges, Methods, and Applications," Computational and Mathematical Methods in Medicine 2015, 1-23 (2015).

[19] Thyreau, B., Sato, K., Fukuda, H. and Taki, Y., "Segmentation of the hippocampus by transferring algorithmic knowledge for large cohort processing," Medical Image Analysis 43, 214-228 (2018).

[20] Focke, N. K., Yogarajah, M., Symms, M. R., Gruber, O., Paulus, W. and Duncan, J. S., "Automated MR image classification in temporal lobe epilepsy," NeuroImage 59(1), 356-362 (2012).

[21] Chupin, M., Hammers, A., Liu, R. S. N., Colliot, O., Burdett, J., Bardinet, E., Duncan, J. S., Garnero, L. and Lemieux, L., "Automatic segmentation of the hippocampus and the amygdala driven by hybrid constraints: Method and validation," Neuroimage 46(3), 749-761 (2009).

[22] Dahi, F., Parsons, M. S., Orlowski, H. L. P., Salter, A., Dahiya, S. and Sharma, A., "Image processing to improve detection of mesial temporal sclerosis in adults," American Journal of Neuroradiology 40(5), 798-801 (2019). 
[23] Strnad, B. S., Orlowski, H. L. P., Parsons, M. S., Salter, A., Dahiya, S. and Sharma, A., "An image processing algorithm to aid diagnosis of mesial temporal sclerosis in children: a case-control study," Pediatric Radiology 50(1), 98-106 (2020).

[24] Lai, C., Guo, S., Cheng, L., Wang, W. and Wu, K., "Evaluation of feature selection algorithms for classification in temporal lobe epilepsy based on MR images," presented at Proceedings of SPIE - The International Society for Optical Engineering, 2017.

[25] Huppertz, H.-J., Wagner, J., Weber, B., House, P. and Urbach, H., "Automated quantitative FLAIR analysis in hippocampal sclerosis,” Epilepsy Research 97(1-2), 146-156 (2011).

[26] Hajiesmaeili, M., Bagherinakhjavanlo, B., Dehmeshki, J. and Ellis, T., "Segmentation of the Hippocampus for Detection of Alzheimer's Disease," Advances in Visual Computing, G. Bebis, R. Boyle, B. Parvin, D. Koracin, C. Fowlkes, S. Wang, M.-H. Choi, S. Mantler, J. Schulze, D. Acevedo, K. Mueller, and M. Papka, Eds., 42-50, Springer, Berlin, Heidelberg (2012).

[27] Rathi, V. P. G. P. and Palani, S., "Brain tumor MRI image classification with feature selection and extraction using linear discriminant analysis," arXiv:1208.2128 [cs] (2012).

[28] Zhou, C., Cheng, Y., Ping, L., Xu, J., Shen, Z., Jiang, L., Shi, L., Yang, S., Lu, Y. and Xu, X., "Support Vector Machine Classification of Obsessive-Compulsive Disorder Based on Whole-Brain Volumetry and Diffusion Tensor Imaging,” Front Psychiatry 9 (2018). 